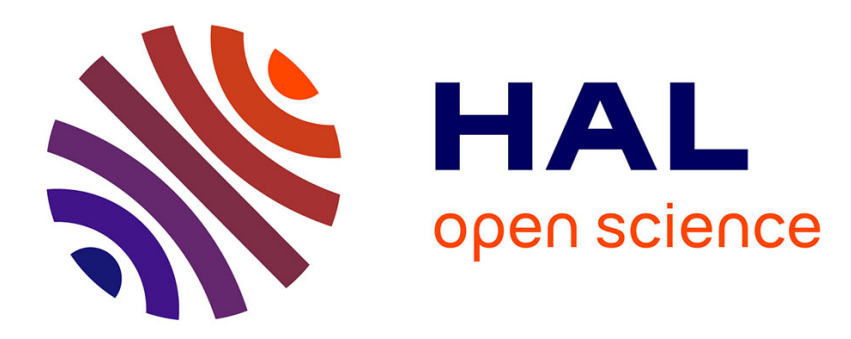

\title{
Relativistic theory of indirect nuclear spin-spin coupling
}

Hubert Ebert

\section{To cite this version:}

Hubert Ebert. Relativistic theory of indirect nuclear spin-spin coupling. Philosophical Magazine, 2008, 88 (18-20), pp.2673-2681. 10.1080/14786430802375659 . hal-00513949

\section{HAL Id: hal-00513949 \\ https://hal.science/hal-00513949}

Submitted on 1 Sep 2010

HAL is a multi-disciplinary open access archive for the deposit and dissemination of scientific research documents, whether they are published or not. The documents may come from teaching and research institutions in France or abroad, or from public or private research centers.
L'archive ouverte pluridisciplinaire $\mathbf{H A L}$, est destinée au dépôt et à la diffusion de documents scientifiques de niveau recherche, publiés ou non, émanant des établissements d'enseignement et de recherche français ou étrangers, des laboratoires publics ou privés. 


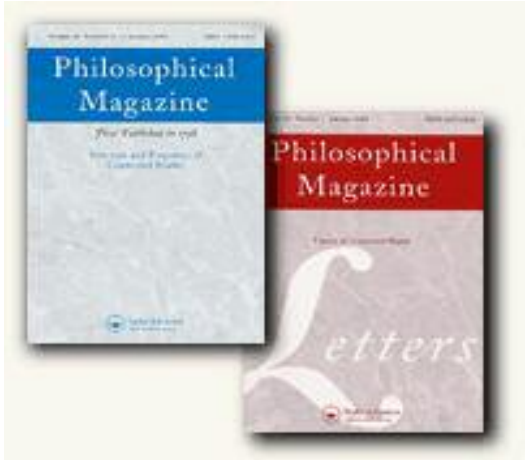

\section{Relativistic theory of indirect nuclear spin-spin coupling}

\begin{tabular}{|r|l|}
\hline Journal: & Philosophical Magazine \& Philosophical Magazine Letters \\
\hline Manuscript ID: & TPHM-08-Jul-0252 \\
\hline Journal Selection: & Philosophical Magazine \\
\hline Date Submitted by the \\
Author: & 14-Jul-2008 \\
\hline Complete List of Authors: & Ebert, Hubert; University of Munich, Chemistry and Biochemistry \\
\hline Keywords: & electronic structure, hyperfine interactions, magnetic properties \\
\hline Keywords (user supplied): & relativistic effects \\
\hline &
\end{tabular}

\section{(s) ScholaroNE" \\ Manuscript Central}


Philosophical Magazine

Vol. 00, No. 00, 21 December 2008, 1-9

\title{
RESEARCH ARTICLE
}

\section{Relativistic theory of indirect nuclear spin-spin coupling}

\author{
H. Ebert* \\ Department Chemie and Biochemie - Physikalische Chemie, Universität München, \\ Butenandtstr. 5-13, D-81377 München, Germany
}

(July 2008)

\begin{abstract}
A fully relativistic theory of indirect nuclear spin-spin coupling is presented that is based on the Green's function formalism. Implementation by the use of multiple scattering or KorringaKohn-Rostoker (KKR) method leads to a very flexible and numerically efficient approach. Results obtained for fcc-Cu are found in full accordance with previous non- or scalar-relativistic calculations and show essentially a behaviour expected from Ruderman-Kittel theory for freeelectron like systems. Results for $\mathrm{Ag}$ and $\mathrm{Au}$ are represented in addition to demonstrate the impact of relativistic effects.
\end{abstract}

\section{Introduction}

In a solid or a molecule nuclear spins are coupled to each other via the direct magnetic dipole-dipole interaction. In addition, there is an indirect coupling nuclear spin-spin that is mediated by the electrons of the system and that can be much larger than the direct one. [1,2] The indirect coupling stems from the perturbation of the electronic system due to the (magnetic) hyperfine interaction with one nucleus that leads to a change in the hyperfine interaction of the electrons with the other nuclei. In general, this mechanism is dealt with by using second order perturbation theory and the conventional decomposition of the hyperfine interaction into a Fermi-contact for s-electrons and a spin-dipolar and orbital term for non-s-electrons, respectively. As the Fermi-contact interaction is in general much stronger than the other ones the indirect nuclear spin-spin coupling is dominated by the so-called Ruderman-Kittel (RK) interaction [3] that corresponds to a Fermicontact electron spin - nuclear spin coupling for both nuclei involved. Adopting a free-electron like description for the underlying electronic structure one is led to a coupling strength of the RK interaction that decays for large inter-nuclear distances $R_{m n}$ in an oscillatory way as $\cos \left(2 k_{F} R_{m n}\right) / R_{m n}^{3}$, with $k_{F}$ being the Fermi vector. In contrast to the RK-interaction the additional coupling terms, that are conventionally called pseudo-dipolar, tensor-tensor and orbital,[1] give rise to an anisotropic coupling between the nuclear spins. As was discussed by Oja et al.[4] this anisotropy reflects the symmetry of the crystal.

Although the theory of indirect nuclear spin-spin coupling has been worked out already in the 1950-ies [1, 3] only very few corresponding numerical investigations on solids can be found in the literature. Only in the 1980-ies, obviously triggered by the observation of nuclear magnetic ordering due to the indirect nuclear spin-spin coupling, [5] the first rigorous calculations have been performed for $\mathrm{Cu}[4,6,7]$ and Ag. [8] While Frisken and Miller [6] considered only the RK interaction, Oja et al.[4]

*Corresponding author. Email: Hubert.Ebert@cup.uni-muenchen.de

ISSN: 1478-6435 print/ISSN 1478-6443 online

(C) 2008 Taylor \& Francis

DOI: $10.1080 / 1478643 \mathrm{YYXxxxxxxx}$

http://www.informaworld.com 
also evaluated the anisotropic coupling terms. In addition, these authors accounted for the influence of scalar-relativistic effects (i.e. without spin-orbit coupling) on the hyperfine interaction. A fully relativistic theory of indirect nuclear spin-spin coupling that includes in particular the effects of spin-orbit coupling and also considers the pseudo-dipolar interactions was worked out by Tterlikkis et al.[9] and applied to the free electron-like alkali metals $\mathrm{Rb}$ and $\mathrm{Cs}$. The influence of many body and also of relativistic effects on the indirect nuclear spin-spin coupling has later on been discussed by Tripathi.[10]

All numerical investigations on the indirect nuclear spin-spin coupling were based so far on an expression obtained by a conventional application of second-order perturbation theory. For an ordered solid this leads to a double k-space integration that is numerically quite hard to handle. In the following we present an alternative formulation that avoids this integration in a natural way by working in a real space representation of the electronic structure and that allows to include all coupling terms in a straight forward way. To account properly for the influence of all relativistic effects on the hyperfine matrix elements as well as on the electronic structure we adapt a fully relativistic formulation.

Our approach will be described in some detail in the next section. This will be followed by a presentation of corresponding results for $\mathrm{Cu}, \mathrm{Ag}$ and $\mathrm{Au}$. The results will be discussed in comparison with available experimental and theoretical data taken from the literature.

\section{Theoretical framework}

\subsection{Electronic structure}

A very powerful and flexible representation of the electronic structure of a solid is supplied by the corresponding electronic Green's function. This applies in particular if local spin density functional theory or an extension to this is adopted as a formal basis to deal with exchange and correlation. Corresponding calculations of the electronic Green's function $G\left(\vec{r}, \vec{r}^{\prime}, E\right)$ can be done in a very reliable way by making use of the multiple scattering or Korringa-Kohn-Rostoker (KKR) formalism. In this case $G\left(\vec{r}, \vec{r}^{\prime}, E\right)$, with the cell centred spatial arguments $\vec{r}$ and $\vec{r}^{\prime}$ located in atomic cell $m$ and $n$, respectively, can be written as:[11, 12]

$$
\begin{aligned}
G\left(\vec{r}, \vec{r}^{\prime}, E\right)= & \sum_{\Lambda \Lambda^{\prime}} Z_{\Lambda}^{m}(\vec{r}, E) \tau_{\Lambda \Lambda^{\prime}}^{m n}(E) Z_{\Lambda^{\prime}}^{n \times}\left(\vec{r}^{\prime}, E\right) \\
& -\sum_{\Lambda}\left[Z_{\Lambda}^{n}(\vec{r}, E) J_{\Lambda}^{n \times}\left(\vec{r}^{\prime}, E\right) \Theta\left(r^{\prime}-r\right)\right. \\
& \left.+J_{\Lambda}^{n}(\vec{r}, E) Z_{\Lambda}^{n \times}\left(\vec{r}^{\prime}, E\right) \Theta\left(r-r^{\prime}\right)\right] \delta_{m n}
\end{aligned}
$$

Within a non-relativistic implementation of the approach the wave functions $Z^{n}$ and $J^{n}$ are the regular and irregular, respectively, solutions to the single site Schrödinger equation for the isolated potential well of the atom at site $n$, that are normalised according to the underlying formulation of scattering theory.[11] To account properly for all relativistic effects $Z^{n}$ and $J^{n}$ are obtained together with the so-called single site t-matrix $t^{n}$ as solutions of the corresponding single site Dirac equation. Accordingly, the index $\Lambda$ stands for the relativistic spin-orbit and magnetic quantum numbers, $\kappa$ and $\mu$, respectively, i.e. $\Lambda=(\kappa, \mu)$.[13] For a 
spherical potential the wave functions $Z^{n}$ and $J^{n}$ are bi-spinors of the form

$$
\Phi_{\Lambda}(\vec{r}, E)=\left(\begin{array}{c}
g_{\Lambda}(r, E) \chi_{\Lambda}(\hat{r}) \\
i f_{\Lambda}(r, E) \chi_{-\Lambda}(\hat{r})
\end{array}\right)
$$

where $g_{\Lambda}$ and $f_{\Lambda}$ are the radial functions connected with the large and small components. Their spin and angular part is represented by the spin-angular functions[13]

$$
\chi_{\Lambda}(\hat{r})=\sum_{m_{s}= \pm 1 / 2} C\left(l \frac{1}{2} j ; \mu-m_{s}, m_{s}\right) Y_{l}^{\mu-m_{s}}(\hat{r}) \chi_{m_{s}},
$$

with $C\left(l \frac{1}{2} j ; \mu-m_{s}, m_{s}\right)$ the Clebsch-Gordan coefficients, $Y_{l}^{m}(\hat{r})$ complex spherical harmonics and $\chi_{m_{s}}$ Pauli spinors. For a non-spherical potential or a spin-polarised system the wave functions can be written as a superposition of bi-spinors with spin-angular character $\Lambda^{\prime}$ and the form as given by Eq. (2).

All multiple scattering events in the system, i.e. hybridisation and band formation, are represented in a self-consistent way by the scattering path operator matrix $\tau^{m n}$, that turns an incoming wave at site $n$ to an outgoing wave at site $m$ with all possible intermediate scattering events accounted for. For a finite system $\tau^{m n}$ can be obtained by inverting the so-called real space KKR-matrix

$$
\tau^{m n}(E)=\left(\left[\underline{\underline{t}}(E)^{-1}-\underline{\underline{G}}^{0}(E)\right]^{-1}\right)^{m n} .
$$

Here $\underline{\underline{G}}^{0}$ is the so-called free-electron Green's function matrix, whose elements are numbered by the site $(n)$ and orbital $(\Lambda)$ indices.[12] The matrix $\underline{\underline{t}}$ is diagonal with respect to the site index and collects the single-site t-matrices $\overline{\bar{t}}^{n}$ of the various atomic sites $n$. Eqs. (1) and (4) supply the basis to use the formalism presented below to calculate the nuclear spin-spin coupling constants for free clusters or molecules. To deal with infinite solids the scattering path matrix $\tau^{m n}$ has to be calculated in an alternative way. For an ordered crystalline solid the multiple scattering equations can be solved by Fourier transformation leading to the following Brillouin-zone integral:

$$
\tau^{m n}(E)=\frac{1}{V_{B Z}} \int_{V_{B Z}} d^{3} k\left[t(E)^{-1}-G(\vec{k}, E)\right]^{-1} e^{i \vec{k}\left(\vec{R}_{m}-\vec{R}_{n}\right)},
$$

with $G(\vec{k}, E)$ the KKR-structure constant matrix and $V_{B Z}$ the volume of the first Brillouin-zone.[11] The numerical effort to calculate this integral can be reduced substantially by making use of the symmetry of the system.[14, 15] In particular one may use the property

$$
\tau^{m^{\prime} n^{\prime}}=U \tau^{m n} U^{-1}
$$

where the (unitary) symmetry operation $U$ connects the distance vectors $\vec{R}_{m n}=$ $\vec{R}_{m}-\vec{R}_{n}$ and $\vec{R}_{m^{\prime} n^{\prime}}=\vec{R}_{m^{\prime}}-\vec{R}_{n^{\prime}}$ via $\vec{R}_{m^{\prime} n^{\prime}}=U \vec{R}_{m n} \cdot[15]$

\subsection{Hyperfine interaction Hamiltonian}

The hyperfine interaction Hamiltonian in its relativistic form represents the coupling of the electronic current density $\vec{j}_{e l}=-e c \vec{\alpha}$ to the vector potential $\vec{A}_{n}$ of the 
nuclear magnetic moment $\vec{\mu}_{n}$ at site $n:[13]$

$$
\begin{aligned}
H_{h f} & =-\frac{1}{c} \vec{j}_{e l} \cdot \vec{A}_{n}(\vec{r}) \\
& =-e \vec{\mu}_{n} \cdot \frac{\vec{\alpha} \times \vec{r}}{r^{3}},
\end{aligned}
$$

where $\vec{\alpha}$ is the vector of Dirac matrices. Due to the r-dependence of the hyperfine operator it is sufficient to restrict the spatial integration to the atomic cell $n$ with volume $\Omega_{n}$ when evaluating the corresponding matrix elements:

$$
\begin{aligned}
M_{\Lambda \Lambda^{\prime}}^{\mathrm{hf} n}(E) & =\int_{\Omega_{n}} d^{3} r Z_{\Lambda}^{n \times}(\vec{r}, E) \mathcal{H}_{\mathrm{hf}}^{n}(\vec{r}) Z_{\Lambda^{\prime}}^{n}(\vec{r}, E) \\
& =-e g_{n} \mu_{\mathrm{N}} \vec{I}_{n} \cdot \int_{\Omega_{n}} d^{3} r Z_{\Lambda}^{n \times}(\vec{r}, E) \frac{\vec{\alpha} \times \vec{r}}{r^{3}} Z_{\Lambda^{\prime}}^{n}(\vec{r}, E) \\
& =C_{n} \vec{I}_{n} \cdot \vec{M}_{\Lambda \Lambda^{\prime}}^{\mathrm{hf} n}(E) .
\end{aligned}
$$

Here we have replaced the nuclear magnetic moment by the relation $\vec{\mu}_{n}=g_{n} \mu_{\mathrm{N}} \vec{I}_{n}$, where $g_{n}$ is the nuclear gyro-magnetic ratio, $\mu_{N}$ is the nuclear Bohr magneton and $\vec{I}_{n}$ is the nuclear spin operator. In addition we used the abbreviation $C_{n}=-e g_{n} \mu_{\mathrm{N}}$. To obtain the various components of the vector matrix element $\vec{M}_{\Lambda \Lambda^{\prime}}^{\text {hf } n}$ it is most convenient to change to a spherical basis that is connected to the Cartesian one by the relations $M^{ \pm}=\mp \sqrt{\frac{1}{2}}\left(M^{x} \pm i M^{y}\right)$ and $M^{z}=M^{0}$. This way one finds:[13, 16]

$$
\left\langle Z_{\Lambda}\left|\frac{(\vec{\alpha} \times \vec{r})_{\lambda}}{r^{3}}\right| Z_{\Lambda^{\prime}}\right\rangle=i\left[\left\langle g_{\Lambda}\left|\frac{1}{r^{2}}\right| f_{\Lambda^{\prime}}\right\rangle+\left\langle f_{\Lambda}\left|\frac{1}{r^{2}}\right| g_{\Lambda^{\prime}}\right\rangle\right] A_{\Lambda \Lambda^{\prime}}^{\lambda}
$$

with the angular matrix elements

$$
A_{\Lambda \Lambda^{\prime}}^{z}=\Delta\left(l 1 \bar{l}^{\prime}\right) \delta_{\mu \mu^{\prime}} i \begin{cases}4 \frac{\mu \kappa}{4 \kappa^{2}-1} & \text { for } \kappa=\kappa^{\prime} \\ +\sqrt{\frac{1}{4}-\left(\frac{\mu}{\kappa-\kappa^{\prime}}\right)^{2}} & \text { for } \kappa=-\kappa^{\prime}-1 \\ -\sqrt{\frac{1}{4}-\left(\frac{\mu}{\kappa-\kappa^{\prime}}\right)^{2}} & \text { for } \kappa=-\kappa^{\prime}+1\end{cases}
$$

and

$$
A_{\Lambda \Lambda^{\prime}}^{ \pm 1}=\Delta\left(l 1 \bar{l}^{\prime}\right) \delta_{\mu \mu^{\prime}-1} \frac{i}{\sqrt{2}} \begin{cases}4 \frac{\kappa}{4 \kappa^{2}-1} \sqrt{\kappa^{2}-\left(\mu+\frac{1}{2}\right)^{2}} & \text { for } \kappa=\kappa^{\prime} \\ -\frac{1}{4 \kappa+1} \sqrt{\left[\kappa+\left(\mu+\frac{1}{2}\right)\right]\left[\kappa+\left(\mu+\frac{3}{2}\right)\right]} & \text { for } \kappa=-\kappa^{\prime}-(\mathbf{1 1}) \\ -\frac{1}{4 \kappa-1} \sqrt{\left[\kappa-\left(\mu+\frac{1}{2}\right)\right]\left[\kappa-\left(\mu+\frac{3}{2}\right)\right]} & \text { for } \kappa=-\kappa^{\prime}+1\end{cases}
$$

where the symbol $\Delta\left(l 1 \bar{l}^{\prime}\right)$ represents the triangle relation[17] that leads to the selection rules $l-l^{\prime}=0\left(\kappa=\kappa^{\prime}\right.$ or $\left.\kappa=-\kappa^{\prime}-1\right)$ or $\left|l-l^{\prime}\right|=2\left(\kappa=-\kappa^{\prime}+1\right)$.

The use of the hyperfine interaction operator as given above properly accounts for the relativistic enhancement of the hyperfine matrix elements that was exam- 
ined and discussed by various authors.[4, 18, 19] However, the operator in Eq. (7) represents the coupling of the total (spin and orbital) electronic current density to the nuclear magnetic moment and therefore does not give the conventional splitting into the Fermi-contact, spin and orbital contribution. Applying a Gordon decomposition [13] of the electronic current density such a decomposition can nevertheless be achieved $[20,21]$ if an appropriate model for the vector potential within the nucleus is set up. Due to the later restriction this decomposition will not be used here. As a consequence, the conventional splitting of the indirect coupling constants into its various parts (see above) cannot be made. Instead, we will separate only the contribution of the s-electrons and will call this RK part.

\subsection{Indirect nuclear spin-spin coupling}

To achieve at an expression for the indirect nuclear spin-spin coupling constants $A_{m n}$ we consider the perturbation of the electronic system due to the hyperfine interaction $\mathcal{H}_{\mathrm{hf}}^{n}$ with a nuclear magnetic moment $\mu_{n}$ at site $n$. Using Dysons's equation the corresponding Green's function $G^{n}$ for the perturbed system is given by

$$
G^{n}\left(\vec{r}, \vec{r}^{\prime}, E\right)=G\left(\vec{r}, \vec{r}^{\prime}, E\right)+\int_{\Omega_{n}} d^{3} r^{\prime \prime} G\left(\vec{r}, \vec{r}^{\prime \prime}, E\right) \mathcal{H}_{\mathrm{hf}}^{n}\left(\vec{r}^{\prime \prime}\right) G\left(\vec{r}^{\prime \prime}, \vec{r}^{\prime}, E\right)
$$

Here we make use of a linear approximation with respect $\mathcal{H}_{\mathrm{hf}}^{n}$ that is well justified and a restriction of the perturbation to the atomic cell around site $n$ (see above, Eq. (7)). Note that the later step does not imply that the perturbation has no impact on the electronic structure in other cells $m \neq n$.

The hyperfine interaction energy for an additional nuclear moment at site $m$ can now be obtained from:[22]

$$
\begin{aligned}
\left\langle\mathcal{H}_{\mathrm{hf}}^{m n}\right\rangle= & -\frac{1}{\pi} \Im \text { Trace } \int^{E_{F}} d E \int_{\Omega_{m}} d^{3} r \mathcal{H}_{\mathrm{hf}}^{m}(\vec{r}) G(\vec{r}, \vec{r}, E) \\
& -\frac{1}{\pi} \Im \operatorname{Trace} \int^{E_{F}} d E \int_{\Omega_{m}} d^{3} r \mathcal{H}_{\mathrm{hf}}^{m}(\vec{r}) \int_{\Omega_{n}} d^{3} r^{\prime} G\left(\vec{r}, \vec{r}^{\prime}, E\right) \mathcal{H}_{\mathrm{hf}}^{n}\left(\vec{r}^{\prime}\right) G\left(\vec{r}^{\prime}, \vec{r}, E\right) \\
= & \left\langle\mathcal{H}_{\mathrm{hf}}^{m}\right\rangle^{(1)}+\left\langle\mathcal{H}_{\mathrm{hf}}^{m n}\right\rangle^{(2)}
\end{aligned}
$$

As above, the hyperfine interaction operator $\mathcal{H}_{\mathrm{hf}}^{m}$ has been restricted to cell $m$. The resulting first term leads obviously to the Knight shift in non-magnetic solids and the static hyperfine field in magnetically ordered solids. The second term, on the other hand, represents the indirect coupling of the nuclear magnetic moments at site $m$ and $n$. Accordingly, only the second term will be considered in the following. Inserting the multiple scattering representation for the Green's function given in 
Eq. (1) one has

$$
\begin{aligned}
\left\langle\mathcal{H}_{\mathrm{hf}}^{m n}\right\rangle^{(2)=}= & \frac{1}{\pi} \Im \int^{E_{F}} d E \sum_{\Lambda \Lambda^{\prime}} \sum_{\Lambda^{\prime \prime} \Lambda^{\prime \prime \prime}} \tau_{\Lambda \Lambda^{\prime}}^{m n}(E) \tau_{\Lambda^{\prime \prime} \Lambda^{\prime \prime \prime}}^{n m}(E) \\
& \int_{\Omega_{m}} d^{3} r Z_{\Lambda^{\prime \prime \prime}}^{m \times}(\vec{r}, E) \mathcal{H}_{\mathrm{hf}}^{m}(\vec{r}) Z_{\Lambda}^{m}(\vec{r}, E) \int_{\Omega_{n}} d^{3} r^{\prime} Z_{\Lambda^{\prime}}^{n \times}\left(\vec{r}^{\prime}, E\right) \mathcal{H}_{\mathrm{hf}}^{n}\left(\vec{r}^{\prime}\right) Z_{\Lambda^{\prime \prime}}^{n}\left(\vec{r}^{\prime}, E\right) \\
= & -\frac{1}{\pi} \Im \int^{E_{F}} d E \sum_{\Lambda \Lambda^{\prime}} \sum_{\Lambda^{\prime \prime} \Lambda^{\prime \prime \prime}} M_{\Lambda^{\prime \prime \prime} \Lambda}^{\mathrm{hf} m}(E) \tau_{\Lambda \Lambda^{\prime}}^{m n}(E) M_{\Lambda^{\prime} \Lambda^{\prime \prime}}^{\mathrm{hf} n}(E) \tau_{\Lambda^{\prime \prime} \Lambda^{\prime \prime \prime}}^{n m}(E) \\
= & -\frac{1}{\pi} \Im \int^{E_{F}} d E \\
& \sum_{\Lambda \Lambda^{\prime}} \sum_{\Lambda^{\prime \prime} \Lambda^{\prime \prime \prime}}\left(C_{m} \vec{I}_{m} \cdot \overrightarrow{\bar{M}}_{\Lambda^{\prime \prime \prime} \Lambda}^{\mathrm{hf} m}(E) \tau_{\Lambda \Lambda^{\prime}}^{m n}(E)\right)\left(C_{m} \vec{I}_{n} \cdot \overrightarrow{\bar{M}}_{\Lambda^{\prime} \Lambda^{\prime \prime}}^{\mathrm{hf} n}(E) \tau_{\Lambda^{\prime \prime} \Lambda^{\prime \prime \prime}}^{n m}(E)\right) \\
= & -\frac{1}{\pi} \Im \int^{E_{F}} d E \\
& \sum_{\Lambda \Lambda^{\prime}} \sum_{\Lambda^{\prime \prime} \Lambda^{\prime \prime \prime}} \vec{I}_{m}\left(C_{m} \vec{M}_{\Lambda^{\prime \prime \prime} \Lambda}^{\mathrm{hf} m}(E) \tau_{\Lambda \Lambda^{\prime}}^{m n}(E)\right) \otimes\left(C_{m} \overrightarrow{\vec{M}}_{\Lambda^{\prime} \Lambda^{\prime \prime}}^{\mathrm{hf} n}(E) \tau_{\Lambda^{\prime \prime} \Lambda^{\prime \prime \prime}}^{n m}(E)\right) \vec{I}_{n}
\end{aligned}
$$

Using the standard form of the indirect nuclear spin-spin coupling Hamiltonian for the total nuclear spin system

$$
\mathcal{H}_{\text {nuc }}=-\frac{1}{2} \sum_{n \neq m} \vec{I}_{n} A_{m n} \vec{I}_{m}
$$

one has finally for the corresponding coupling tensor

$$
\begin{aligned}
A_{m n}= & \frac{1}{2 \pi} C_{m} C_{n} \Im \int^{E_{F}} d E \\
& \sum_{\Lambda^{\prime}} \sum_{\Lambda^{\prime \prime \prime}}\left(\sum_{\Lambda} \vec{M}_{\Lambda^{\prime \prime \prime} \Lambda}^{\mathrm{hfm}}(E) \tau_{\Lambda \Lambda^{\prime}}^{m n}(E)\right) \otimes\left(\sum_{\Lambda^{\prime \prime}} \vec{M}_{\Lambda^{\prime} \Lambda^{\prime \prime}}^{\mathrm{hf}}(E) \tau_{\Lambda^{\prime \prime} \Lambda^{\prime \prime \prime}}^{n m}(E)\right)
\end{aligned}
$$

The resulting expression involves only quantities formulated with respect to real space. Accordingly, it can be applied to molecules as well as to solids. For the later case it requires only to evaluate Brillouin zone integrals of moderate complexity. As Eq. (20) for the coupling tensor is based on the full hyperfine interaction operator, it includes contributions from all electrons and will show an anisotropy according to the symmetry of the solid. As was pointed out by Oja et al.,[4] for a pair of nuclear moments at sites $m$ and $n$ whose distance vector $\vec{R}_{m n}$ is related by a symmetry operation $U$ to that of a pair at $m^{\prime}$ and $n^{\prime}$ by the relation $\vec{R}_{m^{\prime} n^{\prime}}=U \vec{R}_{m n}$ one has for the corresponding coupling tensors

$$
A_{m^{\prime} n^{\prime}}=U A_{m n} U^{-1}
$$

As mentioned above, the use of the full hyperfine Hamiltonian prevents a splitting of the coupling tensor into its RK, pseudo-dipolar etc. parts. Restricting the summations in Eq. (20) to s-electrons, however, one should arrive at a rather good estimate for the RK part $A_{m n}^{R K} \cdot[21]$ 


\section{Results and Discussion}

First results by the use of the scheme sketched above will be presented and discussed in the following. In Fig. 1 results for the indirect nuclear spin-spin coupling constants $A_{m n}$ in the noble metals $\mathrm{Cu}, \mathrm{Ag}$ and $\mathrm{Au}$ are shown as a function of the neighbour distance $R_{m n}$. Obviously, the results follow qualitatively the expected
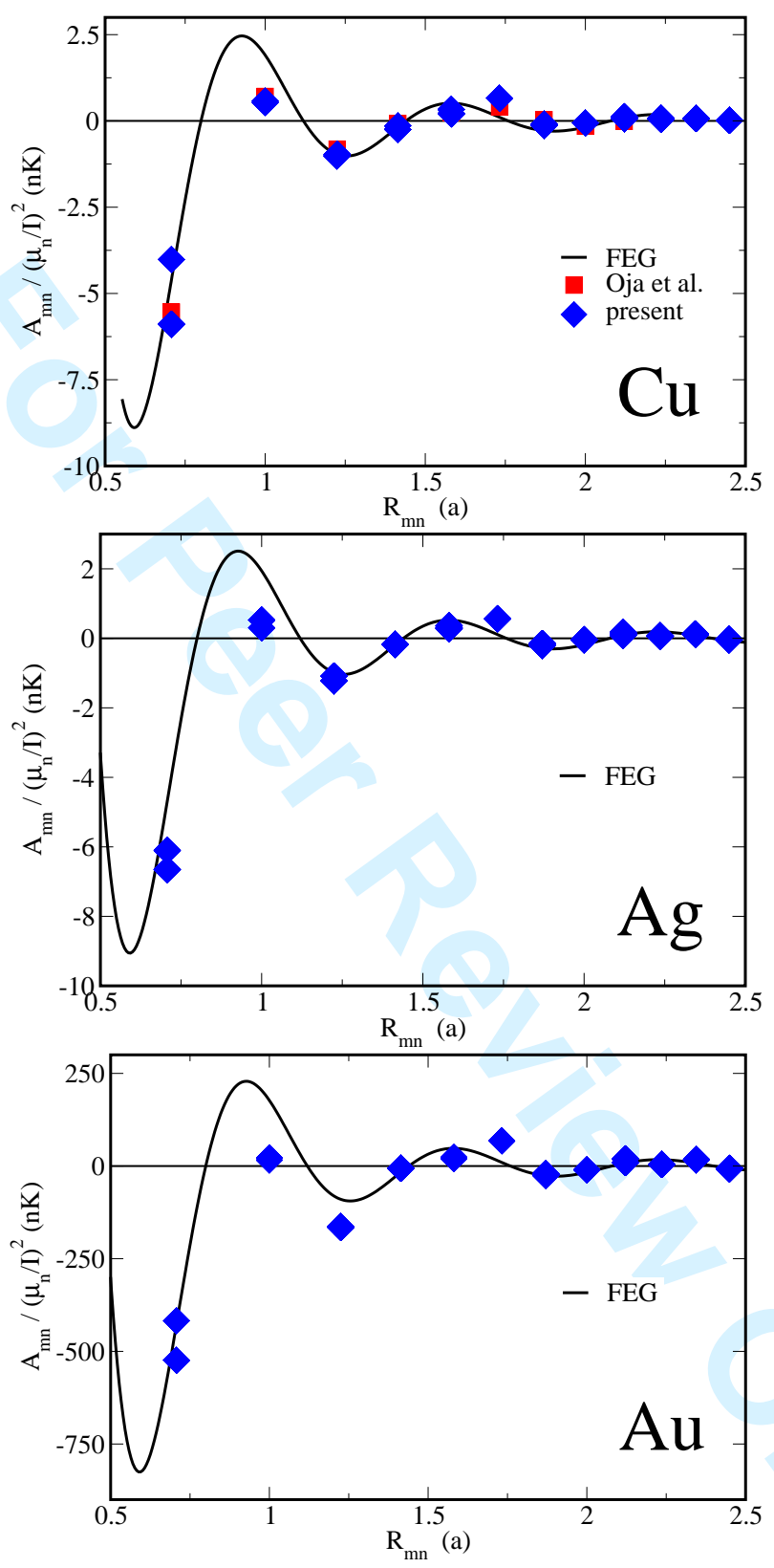

Figure 1. Indirect nuclear spin-spin coupling constants $A_{m n}$ in the noble metals $\mathrm{Cu}, \mathrm{Ag}$ and $\mathrm{Au}$ as a function of the neighbour distance $R_{m n}$. The diamonds represent theoretical results of the present work, while the squares give the isotropic part of $A_{m n}$ for $\mathrm{Cu}$ as calculated by Oja et al.[4] The full lines give the variation of $A_{m n}$ according to the free-electron gas (FEG) description with the amplitude adjusted.

Ruderman-Kittel-like variation with $R_{m n}$ given by $\cos \left(2 k_{F} R_{m n}\right) / R_{m n}^{3}$. Deviations from this simple behaviour are to be assigned to the fact that the Fermi surfaces of the noble metals are not strictly free-electron like. Furthermore there are nonFermi-contact contributions of non-s-electrons that lead to a dependency of $A_{m n}$ not only on $R_{m n}$ but also on the direction $\hat{R}_{m n}$. This can be noticed very clearly in 
few cases. In the top panel of Fig. 1 theoretical results for the isotropical part of $A_{m n}$ due to the Fermi-contact contribution obtained by Oja et al.[4] have been added. These authors applied the conventional approach for their calculation based on perturbation theory together with the scalar-relativistic LAPW (linear augmented plane wave) band structure method and the spin-orbit coupling ignored. Obviously, both ab-initio approaches give results in fairly good agreement with each other.

As discussed by Oja et al.[4] a direct comparison with experimental data can be made in terms of the so-called $R$ and $Q$ parameters, that are defined by

$$
\begin{aligned}
R & =\frac{\sum_{m} A_{m n}^{\mathrm{RK}}}{\mu_{0} \hbar^{2} \gamma_{n} \rho} \\
Q & =\frac{\left[\sum_{m}\left(A_{m n}^{\mathrm{RK}}\right)^{2}\right]^{1 / 2}}{\mu_{0} \hbar^{2} \gamma_{n} \rho}
\end{aligned}
$$

where $\rho$ is the density of the involved nuclei.

The parameter $R$ is meant to reflect the average molecular field of all nuclei $m$ acting on a nucleus at $n$. As table 1 shows, again our result for $R$ agrees fairly well with that calculated by Oja et al.[4] and also with the experimental one given by Ekström et al.,[23] who determined $R$ for $\mathrm{Cu}$ by an NMR experiment on highly polarised nuclear spins. The sign of $R$ found in experiment and properly reproduced

\begin{tabular}{c|l|l||c} 
& \multicolumn{2}{|c||}{ Theory } & Expt \\
\hline & Oja & Present & \\
\hline$R$ & -0.37 & -0.38 & -0.42 \\
& & & \pm 0.05 \\
\hline$Q$ & +0.101 & +0.099 & +0.095 \\
& & & \pm 0.003
\end{tabular}

Table 1. The $R$ - and $Q$-parameters of Cu defined by Eq. (22) and (23) as calculated by Oja et al.[4] and within the present work in comparison with experimental data. [2, 23]

by theory is reflecting the anti-ferromagnetic nature of the indirect nuclear spinspin coupling in $\mathrm{Cu}$.

The second parameter $Q$ defined above is related to the average fluctuating fields and could be determined experimentally in a magic angle spinning NMR experiment.[2] As for $R$ there is very satisfying agreement among the theoretical results as well as with experiment (see table 1).

As indicated above inclusion of non-Fermi-contact contributions to the indirect nuclear spin-spin coupling gives rise to an anisotropy. Our numerical results for $\mathrm{Cu}$ are fully in line with that of Oja et al.[4] indicating that spin-orbit coupling for this light element is not very important. Fig. 1 shows that the ratio of the anisotropic and isotropic parts of the indirect nuclear spin-spin coupling parameters $A_{m n}$ does not increase noticeably when going to heavier elements. In fact the variation of the amplitude of $A_{m n}$ with the atomic number seems to be primarily dominated by the increase of the hyperfine coupling matrix elements as was discussed earlier e.g. in the context of the nuclear spin-lattice relaxation time.[18, 19] 


\section{Summary}

A fully relativistic real space formulation for the indirect nuclear spin-spin coupling has been presented that allows a very straightforward implementation and subsequent interpretation of the results. First applications to the noble metals $\mathrm{Cu}$, $\mathrm{Ag}$ and $\mathrm{Au}$ resulted in a very satisfying agreement with previous theoretical results by Oja et al. as well as with available experimental data.

\section{References}

[1] N. Bloembergen and T.J. Rowland, Nuclear spin exchange in solids: $T l^{203}$ and $T l^{205}$ magnetic resonance in thallium and thallic oxide, Phys. Rev. 97 (1955), p. 1679.

[2] E.R. Andrew, J.L. Carolan, and P.J. Randall, Measurement of the Ruderman-Kittel interaction for copper, Phys. Letters 37A (1971), p. 125.

[3] M.A. Ruderman and C. Kittel, Indirect exchange coupling of nuclear magnetic moments by conduction electrons, Phys. Rev. 96 (1954), p. 99.

[4] A.S. Oja, X.W. Wang, and B.N. Harmon, First-principles study of the conduction-electron-mediated interactions between nuclear spins in copper metal, Phys. Rev. B 39 (1989), p. 4009.

[5] M.T. Huiku, T.A. Jyrkkio, J.M. Kyynarainen, A.S. Oja, and O.V. Lounasmaa, Phase diagram for spontaneous nuclear magnetic ordering in copper, Phys. Rev. Letters 53 (1984), p. 1692.

[6] S.J. Frisken and D.J. Miller, Realistic calculation of the indirect-exchange interaction in metals, Phys. Rev. Letters 57 (1986), p. 2971.

[7] P.A. Lindgard, X.W. Wang, and B.N. Harmon, Calculation of the Ruderman-Kittel interaction and magnetic ordering in copper, J. Magn. Magn. Materials 54-57 (1986), p. 1052.

[8] B.N. Harmon, X.W. Wang, and P.A. Lindgard, Calculation of the Ruderman-Kittel interaction and the nuclear magnetic ordering in silver, J. Magn. Magn. Materials 104-107 (1992), p. 2113.

[9] L. Tterlikkis, S.D. Mahanti, and T.P. Das, Relativistic effects on the hyperfine interactions in alkali metals, Phys. Rev. 178 (1969), p. 630.

[10] G.S. Tripathi, Many-body theory of indirect nuclear interactions, Phys. Rev. B 31 (1985), p. 5143.

[11] P. Weinberger Electron Scattering Theory for Ordered and Disordered Matter, Oxford University Press, Oxford, 1990.

[12] H. Ebert, Fully relativistic band structure calculations for magnetic solids - Formalism and Application, in Electronic Structure and Physical Properties of SolidsH. Dreyssé ed., , Vol. 535 of Lecture Notes in Physics Springer, Berlin, 2000, p. 191.

[13] M.E. Rose Relativistic Electron Theory, Wiley, New York, 1961.

[14] A. Gonis, G.M. Stocks, W.H. Butler, and H. Winter, Local-environment fluctuations and densities of states in substitutionally disordered alloys, Phys. Rev. B 29 (1984), p. 555.

[15] T. Huhne and H. Ebert, Symmetry properties of the scattering path operator for arbitrary translationally invariant systems, Phys. Rev. B 65 (2002), p. 205125/1.

[16] H. Ebert, P. Weinberger, and J. Voitländer, Relativistic formulation for the nuclear-spin-lattice relaxation rate in metallic systems: applications to $A g_{x} P t_{1-x}$, Phys. Rev. B 31 (1985), p. 7566.

[17] M.E. Rose Elementary Theory of Angular Momentum, Wiley, New York, 1961.

[18] P. Pyykkö, E. Pajanne, and M. Inokuti, Hydrogen-like relativistic corrections for electric and magnetic hyperfine integrals, Intern. J. Quantum. Chem. VII (1973), p. 785.

[19] H. Ebert and H. Akai, Consequences of relativity for the hyperfine interactions - with applications to transition metals -, Hyperfine Interactions 78 (1993), p. 361.

[20] N.C. Pyper, Exact relativistic analogues of the non-relativistic hyperfine structure operators. I. Theory, Molecular Physics 64 (1988), p. 933.

[21] M. Battocletti and H. Ebert, Decomposition of the relativistic hyperfine interaction operator - with an application to the ferromagnetic alloy systems bcc-Fe $e_{x} \mathrm{Ni}_{1-x}, f c c-F e_{x} P d_{1-x}$, and fcc-Cox $P t_{1-x}$, Phys. Rev. B 64 (2001), p. 094417.

[22] H. Ebert, P. Strange, and B.L. Györffy, A fully relativistic description of the hyperfine interaction in magnetic systems, Hyperfine Interactions 51 (1989), p. 929

[23] J.P. Ekström, J.F. Jacquinot, M.T. Loponen, J.K. Soini, and P. Kumar, Nuclear spin interaction in copper: NMR at high polarization and in low fields, Physica B 98B (1979), p. 45. 